\section{MAKING MANCHESTER MARVELLOUS THIS MONTH}

At this year's British Dental

Conference and Exhibition in

Manchester from 26-28 April, Digital

Dental will be displaying a whole

range of their latest digital imaging

solutions with packages to suit every practice and specialist discipline.

Products include the Vatech Flex3D,

a cost effective, adaptable and

feature-rich extraoral digital imaging unit. The Dental Directory will be exhibiting some of its huge range of equipment, materials and sundries.

Their latest online catalogue features over 26,000 different professional dental products. Dental accountancy firm accountants4dentists will be available to provide advice on areas ranging from basic bookkeeping to accounts preparation, tax compliance and securing finance. Sister company money4dentists will also have an expert team on-hand to give advice. BKH Healthcare, a newly launched independent dental corporate, will be at the conference to help practices and their teams realise their full potential. BKH provide the infrastructure to simplify management and practice growth. Gain an appreciation of Guru's intuitive, user-friendly format by visiting stand B56. Guru is a standalone software that transforms the way treatment options are communicated to patients. Visit Clark Dental at stand number C48.

Clark Dental are a leading provider of quality surgery design and equipment solutions. Water Pik, Inc. will be on stand D39 at the conference. Delegates to the Water Pik stand will be able to learn more about their highly effective range of water flossers, clinically proven to be twice as effective as traditional string floss at improving gum health. Carestream Dental will be on stand B43 displaying their range of advanced equipment software. The Carestream Dental team will explain how the technology can increase productivity and profitability in practices of all sizes. On stand B30 GSK will be sharing the secrets of Sensodyne Repair Ct Protect with delegates. The toothpaste's NovaMin bioactive technology builds a substantive hydroxyapatite-like layer over exposed dentine and within dentine tubules, to help prevent sensitivity. GC UK will be showcasing G-aenial Universal Flo, the flowable composite restorative material with a unique filler technology, on stand B60. Delegates will also be able to learn about GC's FujiTemp LT, the first glass ionomer provisional luting cement that provides versatility, reliability, durability and ease of use. The team from UCL Eastman Dental Institute will be in Manchester offering clinical presentations in the new demonstration theatre. They will encourage potential participants to obtain expert guidance on postgraduate courses. Henry Schein Minerva are inviting delegates to stand A57 to find out how they can benefit from Face the Challenge 2012. Face the Challenge gives practices who work with Henry Schein Minerva in three practice building areas the opportunity to qualify for fantastic rewards and those who qualify in at least five areas could win a holiday worth thousands of pounds. DPAS can demonstrate savings of up to $£ 10,000$ per year for every 1,000 patients (versus competitor standard charges 2012) on their practice-branded dental plan, for those transferring from competitor products. Learn how you can save with DPAS on stand B5. Straumann will be on stand B75 demonstrating their latest digital solution,

STRAUMANN CARES CADCAM. Straumann's CadentiTero intraoral scanner and digital impression system creates an accurate 3D impression of patients' teeth using a digital scanner. Delegates can try Sirona Dental Systems' CEREC software on stand B64. With intuitive operation, the new version 4.0 of this software is easier to use, allowing you to create bespoke restorations at the chairside.

\section{NEW SAFEGUARDING PROGRAMMES}

EduCare and their safeguarding partner, the NSPCC, have launched two new distance learning programmes to complement their extensive safeguarding range.

Child Neglect is a three-module learning programme which is suitable for anyone who has a basic understanding of child protection. It explores neglect and how it can harm babies, children and young people. It also describes the reasons why children and young people may be neglected and looks at how parental ability and a wide range of different circumstances can all contribute to neglect. Signs and indicators of neglect are given for the key stages of development and the third module focuses on what you should do if you are worried about a child. There is a helpful list to use when seeking advice or making a referral and it concludes with signposts to nation-specific definitions, guidance documents and common assessment frameworks.

Child Sexual Abuse is a four-module programme that is for anyone who works in an organisational setting, including those with responsibility for recruitment. It contains facts about sexual abuse and those who may pose a sexual threat to children. It also gives guidance on how you can create a culture of safety for children within your organisation and there are practical steps to help you promote vigilance amongst staff members. It also contains pointers to good practice in the recruitment and vetting of new staff.

For further information or to purchase a programme, visit www.educare.co.uk.

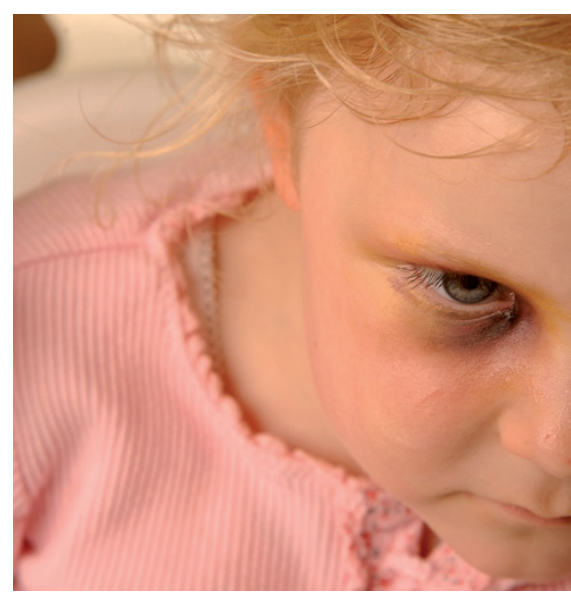

